\title{
Precision analysis of troposphere sensing using GPS single-frequency signals
}

\author{
K. Wang ${ }^{\mathrm{a}, *}$, A. Khodabandeh ${ }^{\mathrm{a}, \mathrm{b}}$, P.J.G. Teunissen ${ }^{\mathrm{a}, \mathrm{c}}$ \\ ${ }^{a}$ GNSS Research Centre, Curtin University of Technology, GPO Box U1987, Perth WA 6845, Australia \\ ${ }^{b}$ Department of Infrastructure Engineering, The University of Melbourne, Melbourne, Australia \\ ${ }^{c}$ Department of Geoscience and Remote Sensing, Delft University of Technology, 2628 CN Delft, The \\ Netherlands
}

\begin{abstract}
Various studies have been performed to investigate the accuracy of troposphere zenith wet delays (ZWDs) determined from GPS. Most of these studies use dual-frequency GPS data of large-scale networks with long baselines to determine the absolute ZWDs. For small-scale networks the estimability of the absolute ZWDs deteriorates due to high correlation between the solutions of the ZWDs and satellite-specific parameters as satellite clocks. However, as relative ZWDs (rZWDs) can always be estimated, irrespective of the size of the network, it is of interest to understand how the large-scale network rZWD-performance of dual-frequency GPS using an ionosphere-float model compares to the small-scale network rZWD-performance of single-frequency GPS using an ionosphere-weighted model. In this contribution such an analysis is performed using undifferenced and uncombined network parametrization modelling. In this context we demonstrate the ionosphere weighted constraints, which allows the determination of the rZWDs independent from signals on the second frequency. Based on an analysis of both simulated and real data, it is found that under quiet ionosphere conditions, the accuracy of the single-frequency determined rZWDs in the ionosphere-weighted network is comparable to that of the large-scale dual-frequency network without ionospheric constraints. Making use of the real data from two baselines of 15 days, it was found that the absolute differences of the rZWDs applying the two strategies are within $1 \mathrm{~cm}$ in over $90 \%$ and $95 \%$ of the time for ambiguity-float and -fixed cases, respectively.
\end{abstract}

Keywords: Troposphere, Zenith Wet Delay (ZWD), Global Positioning System (GPS), Ionosphere, Single-frequency, Dual-frequency

\section{Introduction}

GNSS meteorology (Bevis et al. 1992) provides the possibility to retrieve the temporal and spatial variation of the precipitable water vapour (PWV) and as such is an alternative to other techniques such as radiosondes (Coster et al. 1996), water vapour radiometer (WVR) (Gradinarsky and Elgered 2000), very long baseline interferometry (VLBI) (Coster et al. 1996; Niell et al. 2001) and Doppler orbitography radiopositioning integrated by satellite (DORIS) (Bock

\footnotetext{
${ }^{*}$ Corresponding author

Email addresses: kan.wang@curtin.edu.au (K. Wang), amir.khodabandeh@curtin.edu.au (A. Khodabandeh), p.teunissen@curtin.edu.au (P.J.G. Teunissen)
} 
et al. 2014). The GNSS-based meteorological results are also widely used for weather nowcasting, forecasting and global climate change research (Bevis et al. 1992; Ding et al. 2017).

The accuracy of the GPS-derived PWV are related to that of the wet part of the zenith tropospheric delays, namely the zenith wet delays (ZWDs). Due to the characteristics of water vapour, the ZWDs cannot be a-priori modelled with high accuracy (Resch 1984) and therefore often remain as unknowns in the GNSS observation equations. Depending on the processing strategy, the precision of the a priori information and the correlation with other estimated parameters, the accuracy of the estimated ZWDs can vary from millimetres to centimetres (Dach and Dietrich 2000; Dousa 2001; Ge et al. 2002; Golaszewski et al. 2017; Niell et al. 2001; Rothacher and Beutler 1998). To remove the first-order ionospheric delays, DF-observations are often utilized to form the ionosphere-free linear combination (Ge et al. 2002; Yu et al. 2018) for large-scale network processing. Often also the double-differenced observables are formed to eliminate clock-parameters and hardware biases, and to enable ambiguity resolution (Dach and Dietrich 2000; Ge et al. 2002). Fixing orbit and satellite clock products to known values, using SF receivers, the zenith total delays (ZTDs) can also be determined based on the Precise Point Positioning (PPP) algorithm with the ionospheric delays obtained from the Satellite-specific Epoch-differenced Ionospheric Delay (SEID) model making use of DF signals from surrounding stations (Barindelli et al. 2018; Deng et al. 2011). In this study, we keep all the information in the observational model and make use of the undifferenced and uncombined GPS observation equations (Odijk et al. 2016), so that freedom is left for applying appropriate dynamic models for parameters that otherwise would have been eliminated.

We apply this approach to two type of networks, a DF large-scale network in which no information about the ionospheric delays is provided, and a SF small-scale network based on an ionosphere-weighted model, i.e., in which stochastic spatial constraints are placed on the differential ionospheric delays. The network coordinates processed by AUSPOS (AUSPOS 2018) and orbits, as provided by the international GNSS Services (IGS) (IGS, Dow et al. 2009; IGS 2018; Noll 2010), are given in IGS14 (Figurski and Nykiel 2017) and are assumed known, while for the remaining unknowns estimable parameters are formed to remove the rank deficiencies in the design matrix based on the $S$-system theory (Baarda 1981; Teunissen 1985). When the tropospheric mapping functions for different stations to one satellite are almost the same in small-scale networks, the design matrix becomes almost rank-defect due to the similar structures of the columns of ZWDs and satellite clocks. As a consequence, the absolute ZWD solutions become poorly estimable (Odijk et al. 2016). They are often estimated in large-scale networks including baselines longer than $500 \mathrm{~km}$ (Rocken et al. 1995) or more (Tregoning et al. 1998). However, assuming that within a CORS network one of the station has known ZWDs or PWV by, e.g., connecting with radiosonde or nesting with a DF receiver nearby, the ZWD estimation is only required in relative sense (Rocken et al. 1995) and smaller networks can be utilized. In this study, the relative ZWDs (rZWDs) will be compared between the two networks, i.e., the ones obtained by differencing the absolute ZWDs determined from the DF large network using an ionosphere-float model and those obtained from the SF small network using an ionosphereweighted model. We hereby show the role played by the ionospheric spatial constraints (Odijk 2002). Apart from the real data, simulations are also performed to discuss results under different troposphere and ionosphere conditions. In Section 2, the processing strategies of both approaches are described. With the measurement set up described in Section 3, a detailed analysis of the results is discussed based on both simulated and real data in Section 4. Finally, we summarize the results in Section 5. 


\section{Processing strategy}

In this study, the relative tropospheric ZWDs are estimated separately using a small-scale network with SF GPS signals on L1, denoted as network A and alternatively, also a large-scale network with DF GPS signals on L1 and L2, denoted as network B. The corresponding processing strategies are explained in Sections 2.1 and 2.2.

\subsection{Network A: SF signals}

In GPS network processing, the observed-minus-computed (O-C) terms of the phase $\left(\Delta \phi_{r, j}^{s}\right)$ and the code observations $\left(\Delta p_{r, j}^{s}\right)$ for satellite $s$, receiver $r$ and frequency $j$ can be formulated as follows (Hofmann-Wellenhof et al. 2008; Teunissen and Montenbruck 2017):

$$
\begin{aligned}
& \mathrm{E}\left(\Delta \phi_{r, j}^{s}\right)=g_{r}^{s} \tau_{r}+d t_{r}-d t^{s}-\mu_{j} \iota_{r}^{s}+\delta_{r, j}-\delta_{, j}^{s}+\lambda_{j} a_{r, j}^{s}, \\
& \mathrm{E}\left(\Delta p_{r, j}^{s}\right)=g_{r}^{s} \tau_{r}+d t_{r}-d t^{s}+\mu_{j} \iota_{r}^{s}+d_{r, j}-d_{, j}^{s},
\end{aligned}
$$

where the network stations are CORS in this study with the coordinates assumed to be known. A part of the ZWDs, denoted as $\tau_{r}^{w 0}$, and the zenith hydrostatic delays (ZHDs) are modelled with the Saastamoinen model (Saastamoinen 1972). The remaining ZWDs, denoted as $\tau_{r}$, remain as unknowns and are mapped with the Ifadis mapping function $g_{r}^{s}$ (Ifadis 1986). The total ZWD of receiver $r(r=1, \cdots, n)$ at the time point $t_{i}$, denoted as $\tau_{r}^{w}\left(t_{i}\right)$, can be formulated as:

$$
\tau_{r}^{w}\left(t_{i}\right)=\tau_{r}^{w 0}+\tau_{r}\left(t_{i}\right)
$$

where the modelled ZWD $\tau_{r}^{w 0}$ are assumed to be constant over time for static stations. The receiver and satellite clock errors are represented by $d t_{r}$ and $d t^{s}$, respectively, and the ionospheric delays on the reference frequency $f_{1}$ are denoted by $\iota_{r}^{s}$ and multiplied with the coefficient $\mu_{j}=$ $f_{1}^{2} / f_{j}^{2}$. The receiver and satellite phase hardware delays $\delta_{r, j}$ and $\delta_{, j}^{s}$ exist for each frequency $j$. The same applies also for the receiver and satellite code hardware delays $d_{r, j}$ and $d_{, j}^{s}$. The phase ambiguities $a_{r, j}^{s}$, which are given in cycles, are multiplied with the corresponding wavelength $\lambda_{j}$. $\mathrm{E}(\cdot)$ denotes expectation operator in this contribution. For the small-scale SF network A with the largest inter-station distance of around $30 \mathrm{~km}$, we make use of the ionosphere-weighted scenario by constraining the between-receiver ionospheric delays with distance-dependant weights (Odijk 2002):

$$
\mathrm{E}\left(d \iota_{r}^{s}\right)=\iota_{r}^{s}-\iota_{1}^{s}, \quad r \neq 1,
$$

where $d \iota_{r}^{s}$ are pseudo-observations with sample values of zero, and the weight increases with decreasing distance (see Appendix).

To remove the rank deficiencies in the design matrix, instead of the original unknown parameters in Eqs. 1 and 2, estimable parameters are formed based on $S$-system theory (Baarda 1981; Teunissen 1985). The satellite hardware biases and the ambiguities are assumed to be timeconstant, and the receiver hardware biases and the ZWD increments are assumed to be linked in time with a random-walk process. The O-C terms for phase and code observations (Eqs. 1 and 2) and the spatial ionospheric constraints (Eq. 4) can be reformulated with the estimable parameters $\tilde{\tau}_{r}, d \tilde{t}_{r}, d \tilde{t}^{s}, \tilde{\iota}_{r}^{s}, \tilde{\delta}_{r, j}, \tilde{\delta}_{, j}^{s}, \tilde{d}_{r, j}$ and $\tilde{a}_{r, j}^{s}$ given in Table 1 (Wang et al. 2018):

$$
\begin{aligned}
\mathrm{E}\left(\Delta \phi_{r, j}^{s}\right) & =g_{r}^{s} \tilde{\tau}_{r}+d \tilde{t}_{r}-d \tilde{t}^{s}-\mu_{j} \tilde{\iota}_{r}^{s}+\tilde{\delta}_{r, j}-\tilde{\delta}_{, j}^{s}+\lambda_{j} \tilde{a}_{r, j}^{s}, \\
\mathrm{E}\left(\Delta p_{r, j}^{s}\right) & =g_{r}^{s} \tilde{\tau}_{r}+d \tilde{t}_{r}-d \tilde{t}^{s}+\mu_{j} \tilde{\iota}_{r}^{s}+\tilde{d}_{r, j}, \\
\mathrm{E}\left(d \iota_{r}^{s}\right) & =\tilde{\iota}_{r}^{s}-\tilde{\iota}_{1}^{s}, \quad r \neq 1 .
\end{aligned}
$$


Table 1. Estimable parameters in Eqs. 5 and 6 in single-frequency ionosphere-weighted scenario (Wang et al. 2018). The satellite hardware biases and ambiguities are assumed to be constant, and the receiver hardware biases and ZWD increments are assumed to be linked in time (SF Network A)

\begin{tabular}{ll}
\hline Parameter & Interpretation \\
\hline$\tilde{\tau}_{r \neq 1}\left(t_{1}\right)$ & $\tau_{r}\left(t_{1}\right)-\tau_{1}\left(t_{1}\right)$ \\
$\tilde{\tau}_{r}\left(t_{i>1}\right)$ & $\tau_{r}\left(t_{i}\right)-\tau_{1}\left(t_{1}\right)$ \\
$d \tilde{t}_{r \neq 1}\left(t_{i}\right)$ & $d t_{1 r}\left(t_{i}\right)+d_{1 r, j}\left(t_{1}\right)$ \\
$d \tilde{t}^{s}\left(t_{i}\right)$ & $d t^{s}\left(t_{i}\right)+d_{, j}^{s}-\left(d t_{1}\left(t_{i}\right)+d_{1, j}\left(t_{1}\right)\right)-g_{1}^{s}\left(t_{i}\right) \tau_{1}\left(t_{1}\right)-\mu_{j} \iota_{1}^{s}\left(t_{1}\right)$ \\
$\tilde{\iota}_{r}^{s}\left(t_{i}\right)$ & $\iota_{r}^{s}\left(t_{i}\right)-\iota_{1}^{s}\left(t_{1}\right),\left\{\begin{array}{l}r \neq 1, i=1 \\
\forall r, i>1\end{array}\right.$ \\
$\tilde{\delta}_{r, j}\left(t_{i}\right)$ & $\delta_{r, j}\left(t_{i}\right)-\delta_{1, j}\left(t_{1}\right)-d_{1 r, j}\left(t_{1}\right)+\lambda_{j} a_{1 r, j}^{1},\left\{\begin{array}{l}r \neq 1, i=1 \\
\forall r, i>1\end{array}\right.$ \\
$\tilde{\delta}_{\tilde{j}_{j}}^{s}$ & $\delta_{, j}^{s}-\delta_{1, j}\left(t_{1}\right)-\left(d_{, j}^{s}-d_{1, j}\left(t_{1}\right)\right)+2 \mu_{j} \iota_{1}^{s}\left(t_{1}\right)-\lambda_{j} a_{1, j}^{s}$ \\
$\tilde{d}_{r, j}\left(t_{i>1}\right)$ & $d_{r, j}\left(t_{i}\right)-d_{r, j}\left(t_{1}\right)$ \\
$\tilde{a}_{r \neq 1, j}^{s \neq 1}$ & $a_{1 r, j}^{s}-a_{1 r, j}^{1}$ \\
\hline$S$-basis & $\tau_{1}\left(t_{1}\right), d t_{1}\left(t_{i}\right), \delta_{1, j}\left(t_{1}\right), d_{r, j}\left(t_{1}\right), d_{, j}^{s}, \iota_{1}^{s}\left(t_{1}\right), a_{1, j}^{s}, a_{r, j}^{1}$ \\
\hline
\end{tabular}

The reference receiver and satellite are denoted with the subindex and superindex 1, respectively. The between-receiver (receiver $r$ and the reference receiver) and between-satellite (satellite $s$ and the reference satellite) differences are denoted with $(\cdot)_{1 r}=(\cdot)_{r}-(\cdot)_{1}$ and $(\cdot)^{1 s}=(\cdot)^{s}-(\cdot)^{1}$, respectively.

The Precise Point Positioning - Real-time Kinematic (PPP-RTK) technique enables the integer ambiguity resolution in PPP (Teunissen and Khodabandeh 2015). Our processing is performed in a Kalman filter with the Curtin PPP-RTK Software (Nadarajah et al. 2018; Odijk et al. 2017; Wang et al. 2017). The process noise of the time-linked parameters are listed in Table 2 with s representing second. The parameters not listed are estimated as unlinked parameters in time. As a consequence of the process noise of the original parameters listed in Table 2, the corresponding estimable parameters also share the same values of the system noise, i.e., for $\tilde{\tau}_{r} 0.1 \mathrm{~mm} / \sqrt{\mathrm{s}}$, for $\tilde{\delta}_{r, j}$ and $\tilde{d}_{r, j} 1 \mathrm{~cm} / \sqrt{\mathrm{s}}$, and $\tilde{\delta}_{, j}^{s}, \tilde{a}_{r \neq 1, j}^{s \neq 1}$ are assumed to be constant. The zenith-referenced a priori standard deviations for phase $\left(\sigma_{\phi}^{0}\right)$ and code observations $\left(\sigma_{p}^{0}\right)$ are set to be $1.5 \mathrm{~mm}$ and $1.5 \mathrm{dm}$, respectively. The variances of the phase $\left(\sigma_{\phi}^{2}\right)$ and code observations $\left(\sigma_{p}^{2}\right)$ with the elevation angle $e$ are calculated as (Dach et al. 2015):

$$
\sigma_{\phi}^{2}=\frac{\left(\sigma_{\phi}^{0}\right)^{2}}{\sin ^{2} e}, \quad \sigma_{p}^{2}=\frac{\left(\sigma_{p}^{0}\right)^{2}}{\sin ^{2} e} .
$$

Based on the estimated $\hat{\tilde{\tau}}_{r}$, the rZWD $\hat{\tau}_{1 r}^{w A}$ and its dispersion $\mathrm{D}\left[\hat{\tau}_{1 r}^{w A}\right]$ can be obtained for the SF network A:

$$
\begin{aligned}
\hat{\tau}_{1 r}^{w A} & =\tau_{1 r}^{w 0}+\hat{\tilde{\tau}}_{r}-\hat{\tilde{\tau}}_{1}, \\
D\left[\hat{\tau}_{1 r}^{w A}\right] & =D_{n}^{T} Q_{\hat{\tilde{\tau}} \hat{\tilde{\tau}}} D_{n},
\end{aligned}
$$


Table 2. Process noise of the parameters in the Kalman filter. Parameters not listed are estimated as unlinked parameters in time

\begin{tabular}{cc}
\hline Parameter & Process noise \\
\hline$\tau_{r}\left(t_{i}\right)$ & $0.1 \mathrm{~mm} / \sqrt{\mathrm{s}}$ \\
$\delta_{r, j}\left(t_{i}\right), d_{r, j}\left(t_{i}\right)$ & $1 \mathrm{~cm} / \sqrt{\mathrm{s}}$ \\
$\delta_{, j}^{s}\left(t_{i}\right), d_{, j}^{s}\left(t_{i}\right)$ & 0 \\
$a_{r, j}^{s}\left(t_{i}\right)$ & 0 \\
\hline
\end{tabular}

where $Q_{\hat{\tilde{\tau}} \hat{\tilde{\tau}}}$ represents the variance-covariance matrix of the estimated ZWDs from the SF network A. The term $D_{n}^{T}$ is the differencing operator with $D_{n}^{T}=\left[-e_{n-1}, I_{n-1}\right]$, where $e_{n-1}$ and $I_{n-1}$ denote vector of ones with the length $n-1$ and identity matrix with the size $n-1$, respectively. In this study, the modelled rZWDs $\tau_{1 r}^{w 0}$ are assumed to be non-random.

\subsection{Network B: DF signals}

For comparison purposes, the rZWDs are also calculated using DF GPS signals from a largescale network B with the inter-station distance up to around $757 \mathrm{~km}$. The DF Network B contains the reference station and several other stations of the SF network A. For each of these stations, in simulations, it is assumed that receivers connected to different antennas located at the same positions are used for data tracking in these two networks.

For the large-scale DF network B, the ionosphere-float model is used, i.e., no spatial constraint is put on the between-receiver ionospheric delays. Compared to the SF network A, it is now possible to estimate the absolute ZWD increments of each network station. The O-C terms and the new estimable parameters $\tilde{\tilde{\tau}}_{r}, d \tilde{\tilde{t}}_{r}, d \tilde{\tilde{t}}^{s}, \tilde{\tilde{\iota}}_{r}^{s}, \tilde{\tilde{\delta}}_{r, j}, \tilde{\tilde{\delta}}_{, j}^{s}, \tilde{\tilde{d}}_{r, j}, \tilde{\tilde{d}}_{, j}^{s}$ and $\tilde{\tilde{a}}_{r, j}^{s}$ for DF ionosphere-float scenario are given in Eqs. 11, 12 and Table 3 (Wang et al. 2017) with the same dynamic models used as in Section 2.1:

$$
\begin{aligned}
& \mathrm{E}\left(\Delta \phi_{r, j}^{s}\right)=g_{r}^{s} \tilde{\tilde{\tau}}_{r}+d \tilde{\tilde{t}}_{r}-d \tilde{\tilde{\tilde{t}}}^{s}-\mu_{j} \tilde{\tilde{\iota}}_{r}^{s}+\tilde{\tilde{\delta}}_{r, j}-\tilde{\tilde{\tilde{\delta}}}_{, j}^{s}+\lambda_{j} \tilde{\tilde{a}}_{r, j}^{s} \\
& \mathrm{E}\left(\Delta p_{r, j}^{s}\right)=g_{r}^{s} \tilde{\tilde{\tau}}_{r}+d \tilde{\tilde{t}}_{r}-d \tilde{\tilde{t}}^{s}+\mu_{j} \tilde{\tilde{l}}_{r}^{s}+\tilde{\tilde{d}}_{r, j}-\tilde{\tilde{d}}_{, j}^{s} .
\end{aligned}
$$

Using the modelled ZWDs and the estimated absolute ZWD increments for each station, the rZWDs $\hat{\tau}_{1 r}^{w B}$ can be obtained from the DF network B:

$$
\begin{aligned}
\hat{\tau}_{1 r}^{w B} & =\tau_{1 r}^{w 0}+\hat{\tilde{\tilde{\tau}}}_{r}-\hat{\tilde{\tilde{\tau}}}_{1}, \\
D\left[\hat{\tau}_{1 r}^{w B}\right] & =D_{n}^{T} Q_{\hat{\tilde{\tilde{\tau}}} \hat{\tilde{\tilde{\tau}}}} D_{n},
\end{aligned}
$$

where $Q_{\hat{\tilde{\tilde{\tau}}} \tilde{\tilde{\tau}}}$ represents the variance-covariance matrices of $\hat{\tilde{\tau}}_{r}$ from the DF network B. The data analysis and comparison of the results using networks A and B are discussed in Section 4.

\section{Data selection}

As shown in Figure 1, the SF network A consists of 7 stations in Perth, Australia marked in yellow. The largest inter-station distance is around $30.0 \mathrm{~km}$, and the station CUT0 was used as the reference station. The real observation data of the SF network A were provided by 
Table 3. Estimable parameters in Eqs. 11 and 12 in dual-frequency ionosphere-float scenario (Wang et al. 2017). The satellite hardware biases and ambiguities are assumed to be constant, and the receiver hardware biases and ZWD increments are assumed to be linked in time (DF Network B)

\begin{tabular}{l|l}
\hline Parameter & Interpretation \\
\hline$\tilde{\tilde{\tau}}_{r}\left(t_{i}\right)$ & $\tau_{r}\left(t_{i}\right)$ \\
$d \tilde{\tilde{t}}_{r \neq 1}\left(t_{i}\right)$ & $d t_{1 r}\left(t_{i}\right)+d_{1 r, I F}\left(t_{1}\right)$ \\
$\tilde{\tilde{t}}^{s}\left(t_{i}\right)$ & $d t^{s}\left(t_{i}\right)+d_{, I F}^{s}-\left(d t_{1}\left(t_{i}\right)+d_{1, I F}\left(t_{1}\right)\right)$ \\
$\tilde{\tilde{l}}_{r}^{s}\left(t_{i}\right)$ & $\iota_{r}^{s}\left(t_{i}\right)+d_{r, G F}\left(t_{1}\right)-d_{, G F}^{s}$ \\
$\tilde{\tilde{\delta}}_{r \neq 1, j}\left(t_{i}\right)$ & $\delta_{r, j}\left(t_{i}\right)-\delta_{1, j}\left(t_{1}\right)+\mu_{j} d_{1 r, G F}\left(t_{1}\right)-d_{1 r, I F}\left(t_{1}\right)+\lambda_{j} a_{1 r, j}^{1}$ \\
$\tilde{\delta}_{1, j}\left(t_{i>1}\right)$ & $\delta_{1, j}\left(t_{i}\right)-\delta_{1, j}\left(t_{1}\right)$ \\
$\tilde{\delta}_{\tilde{j}_{j}}^{s}$ & $\delta_{, j}^{s}+\mu_{j}\left(d_{, G F}^{s}-d_{1, G F}\left(t_{1}\right)\right)-\left(d_{, I F}^{s}-d_{1, I F}\left(t_{1}\right)\right)-\delta_{1, j}\left(t_{1}\right)-\lambda_{j} a_{1, j}^{s}$ \\
$\tilde{d}_{r, j=1,2}\left(t_{i>1}\right)$ & $d_{r, j}\left(t_{i}\right)-d_{r, j}\left(t_{1}\right)$ \\
$\tilde{a}_{r \neq 1, j}^{s \neq 1}$ & $a_{1 r, j}^{s}-a_{1 r, j}^{1}$ \\
\hline$S$-basis & $d t_{1}\left(t_{i}\right), \delta_{1, j}\left(t_{1}\right), d_{r, j=1,2}\left(t_{1}\right), d_{, j=1,2}^{s}, a_{1, j}^{s}, a_{r, j}^{1}$ \\
\hline \multicolumn{1}{|c|}{$(\cdot)_{, I F}=\frac{1}{\mu_{2}-\mu_{1}}\left[\mu_{2}(\cdot)_{, 1}-\mu_{1}(\cdot)_{, 2}\right] ; \quad(\cdot)_{, G F}=\frac{1}{\mu_{2}-\mu_{1}}\left[(\cdot)_{, 2}-(\cdot)_{, 1}\right]$}
\end{tabular}

Geoscience Australia (GA, Geoscience Australia 2018), RTKnetwest (RTKnetwest 2017) and the GNSS Research Centre, Curtin University (Curtin GNSS Research Centre 2018). The sampling interval of the data is $30 \mathrm{~s}$, and the elevation mask is set to be 10 degrees. The International GNSS Service (IGS) final orbits (Dow et al. 2009; IGS 2018; Noll 2010) were used for the processing. The coordinates of the stations in both networks were processed with AUSPOS (AUSPOS 2018) and are assumed to be known. Apart from that, the daily rainfall data were collected by 5 meteorological stations marked with white 4-digit numbers in Figure 1 (Bureau of Meteorology 2018). As Perth is a city with a long-term annual average sunshine of more than 8 h per day (Paddenburg 2017), to evaluate the ZWDs under different humidity conditions, 15 different days in June 2017 with different rainfall amounts and with the observation data available were used for the processing. The daily rainfall of the stations STIG, SLTP and CUT0 were interpolated using the data collected from the 5 meteorological stations. The interpolated daily rainfall amounts are shown in Figure 2 in millimetres.

For processing with the small-scale SF network A, simulated and real SF GPS singals on L1 were used to calculate the rZWDs between stations STIG, SLTP and the reference station CUT0. The distances between stations STIG and CUT0 and between stations SLTP and CUT0 amount to around 17 and $3 \mathrm{~km}$, respectively. The number of the GPS satellites above the elevation mask of 10 degrees and the Precision Dilution of Position (PDOP) are illustrated for the reference station CUT0 in Figure 3 on DOY 173, 2017.

For processing using DF GPS signals on L1 and L2, as shown in Figure 4, a larger network B of 13 stations with the inter-station distances up to $757 \mathrm{~km}$ was used. The DF network B contains the stations CUT0, SLTP and STIG in the SF network A, and in simulations it is assumed that for each of these three stations receivers connected to different antennas located at the same positions were used in networks A and B. For real data processing, only for station CUT0, two different receivers connected to the same antenna were used for processing of networks A and B separately. The real observation data of the DF network B were provided by GA 


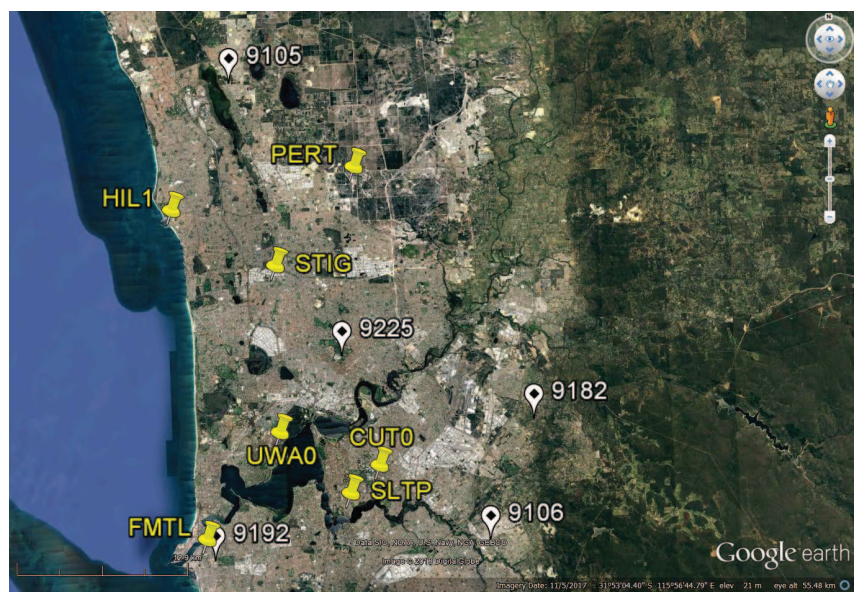

Fig. 1. SF Network A in Perth, Australia with the largest inter-station distance of $30 \mathrm{~km}$. The meteorological stations are marked with white 4-digit numbers. Map data: Google, Data SIO, NOAA, U.S. Navy. NGA. GEBCO, Image (C)2018 DigitalGlobe (Google Earth 2018a)

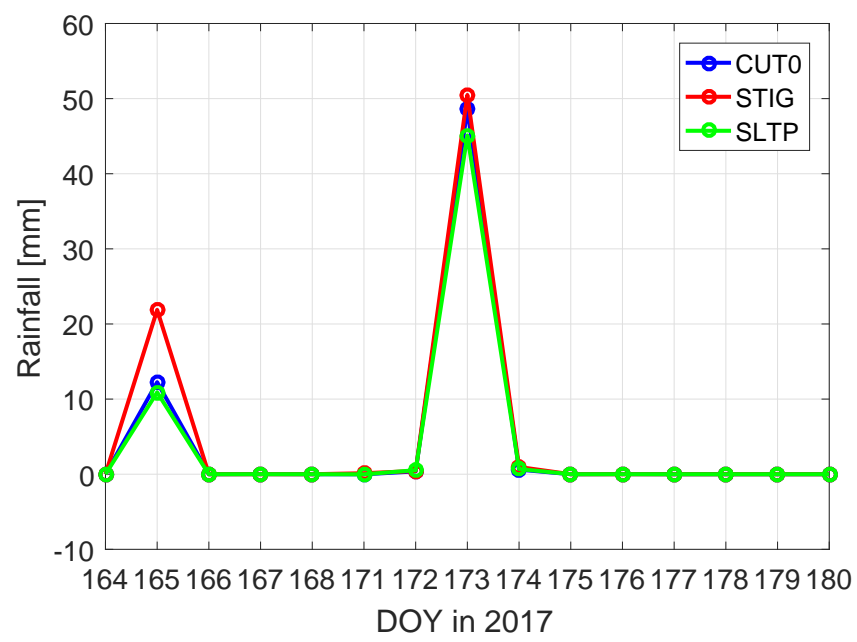

Fig. 2. Interpolated daily rainfall amounts for stations CUT0, STIG and SLTP in 15 days of June 2017. DOY is short for day of year 


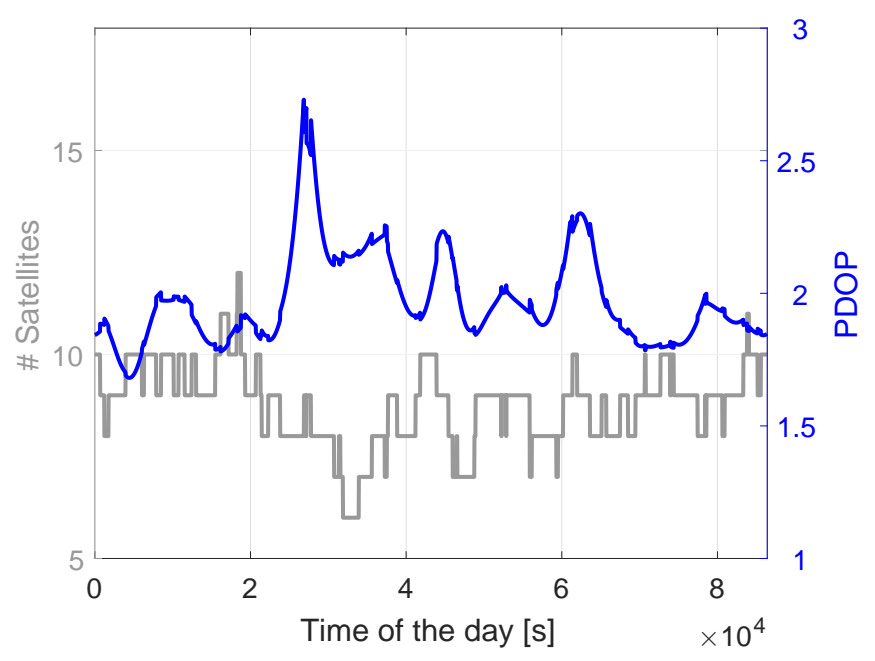

Fig. 3. Number of the GPS satellites above the elevation mask of 10 degrees and the PDOP values for station CUT0 on DOY 173, 2017

(Geoscience Australia 2018), RTKnetwest (RTKnetwest 2017) and the GNSS Research Centre, Curtin University (Curtin GNSS Research Centre 2018). The same 15 days in June 2017 were used for real data analysis in the DF network B as in the SF network A.

\section{Analysis of the results}

In this contribution, both simulated and real data were processed and analysed using solutions from both networks. The results are illustrated and discussed in the next two sub-sections. By computing the daily solutions, the first hour was considered as the filter initialization phase, and the solutions within the first hour of each day were therefore not included in the analysis.

\subsection{Simulated data}

In this sub-section, SF and DF O-C terms were simulated for stations in both the networks A and B. The simulated phase $\left(\Delta \breve{\phi}_{r, j}^{s}\right)$ and code O-C terms $\left(\Delta \breve{p}_{r, j}^{s}\right)$ were formulated with:

$$
\begin{aligned}
\Delta \breve{\phi}_{r, j}^{s} & =g_{r}^{s} \breve{\tau}_{r}-\mu_{j} \breve{\iota}_{r}^{s}+\breve{\delta}_{r, j}, \\
\Delta \breve{p}_{r, j}^{s} & =g_{r}^{s} \breve{\tau}_{r}+\mu_{j} \breve{\iota}_{r}^{s}+\breve{d}_{r, j},
\end{aligned}
$$

where $\breve{\delta}_{r, j}$ and $\breve{d}_{r, j}$ represented the simulated receiver phase and code hardware biases, respectively. Based on their dynamic models set in the Kalman filter (Table 2), they were generated as random-walk noise with the standard deviation of epoch-to-epoch differences amounting to $1 \mathrm{~cm} / \sqrt{\mathrm{s}} \cdot \sqrt{\Delta t}$, where $\Delta t$ denotes the sampling interval of $30 \mathrm{~s}$ in this study. The terms $\breve{\tau}_{r}$ and $\breve{\iota}_{r}^{s}$ denote the simulated ZWDs (after removing the modelled part $\tau_{r}^{w 0}$ ) and ionospheric delays on L1, respectively. Based on the ionosphere-weighted model described in Appendix, the ionospheric delays in the SF network A were simulated with variance-covariance matrix of $\frac{1}{\sin ^{2}\left(\bar{e}^{s}\right)} \cdot \frac{\sigma_{t}^{2}}{2} \cdot Q_{r r}$ for satellite $s$ (Eq. A.3 in Appendix). In the DF network B, the ionospheric delays were estimated as temporally and spatially unlinked parameters and were set as zeros in the simulations. The term $\breve{\tau}_{r}$ was simulated as a constant (here $5 \mathrm{~cm}$ ) added by a random-walk noise with the standard deviation of the epoch-to-epoch differences amounting to $s_{\tau} \sqrt{\Delta t}$. The term $s_{\tau}$ denotes the process noise of the simulated ZWDs, and the corresponding process noise 


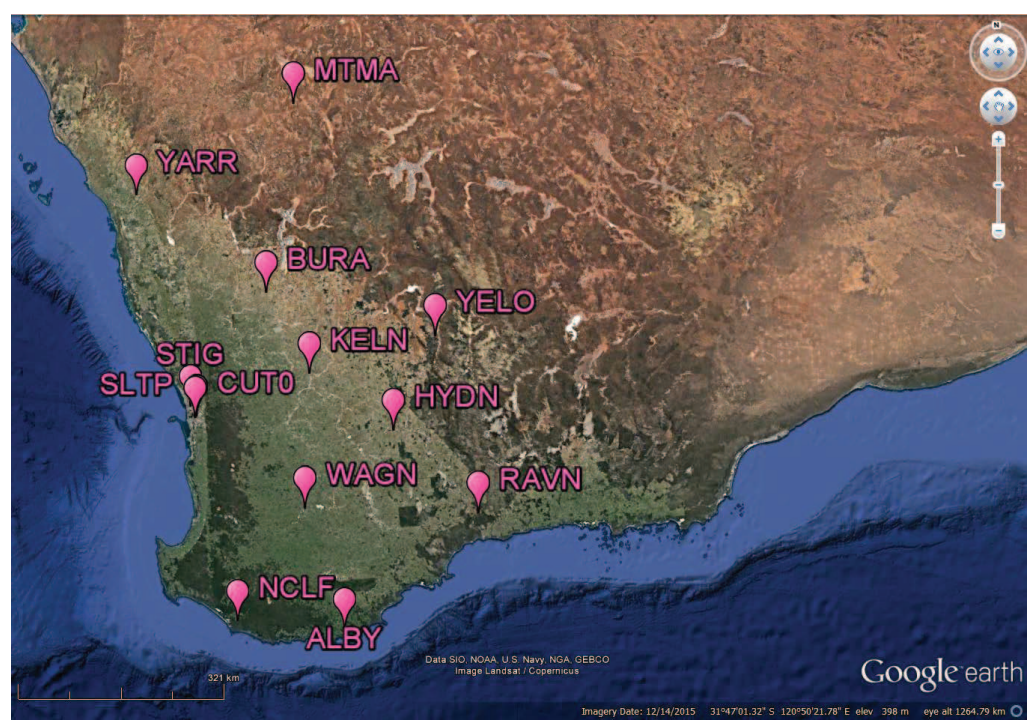

Fig. 4. DF Network B in Western Australia with the largest inter-station distance of around $757 \mathrm{~km}$. Map data: Google, Data SIO, NOAA, U.S. Navy. NGA. GEBCO, Image Landsat/Copernicus (Google Earth 2018b)

for ZWDs set in the dynamic model of the Kalman filter changes also with this pre-defined $s_{\tau}$. The simulated $\breve{\tau}_{r}$ for stations CUT0, STIG and SLTP are the same in both networks, and the process noise of the ZWDs $s_{\tau}$ varies from 0.05 to $0.3 \mathrm{~mm} / \sqrt{\mathrm{s}}$ in the simulations to model different ZWD variations under different weather conditions. We remark that the simulated time series of ZWDs for each station is only one realization of the random-walk signals. The same applies also for the time series of the receiver hardware biases.

The rZWDs and their variance matrices for the two baselines, i.e., baseline CUT0-SLTP with a length of around $3 \mathrm{~km}$ and baseline CUT0-STIG with a length of around $17 \mathrm{~km}$ were computed as described in Section 2 based on the SF signals from network A and DF signals from network B (Eqs. 9, 10, 13 and 14). Figure 5 shows the estimated rZWDs deviated from their true values, which are denoted with

$$
\begin{aligned}
& \Delta \hat{\tau}_{1 r}^{w A}=\hat{\tau}_{1 r}^{w A}-\breve{\tau}_{1 r}^{w}, \\
& \Delta \hat{\tau}_{1 r}^{w B}=\hat{\tau}_{1 r}^{w B}-\breve{\tau}_{1 r}^{w},
\end{aligned}
$$

for networks A and B, respectively. The blue and gray lines represent the deviations and the 95\% formal confidence interval in ambiguity-float case, while the green and black lines illustrate their counterparts in ambiguity-fixed case. For the ambiguity-fixed scenario, partial ambiguity resolution with a pre-defined success rate of $99.9 \%$ was enabled. The process noise of ZWDs $s_{\tau}$ was set to be $0.1 \mathrm{~mm} / \sqrt{\mathrm{s}}$, and for ionosphere-weighted model in SF network A the coefficients $l_{\max }$ (Eq. A.2) and $\sigma_{\iota}$ (Eq. A.3) were set to be $30 \mathrm{~km}$ and $5 \mathrm{~cm}$, respectively. The empirical standard deviations of $\Delta \hat{\tau}_{1 r}^{w A}$ and $\Delta \hat{\tau}_{1 r}^{w B}$ and the average formal standard deviations of $\hat{\tau}_{1 r}^{w A}$ (Eq. 10) and $\hat{\tau}_{1 r}^{w B}$ (Eq. 14) are also listed in Table 4 for both baselines in ambiguity-float and -fixed cases, respectively.

From Figure 5 and Table 4 we see that the empirical and formal solutions correspond well with each other, which shows again the correspondence between the simulations and the model we used. We remark that after the first hour of initialization phase, which was not used for the data analysis, the ambiguity-float solutions converge almost to the same level as the ambiguity- 

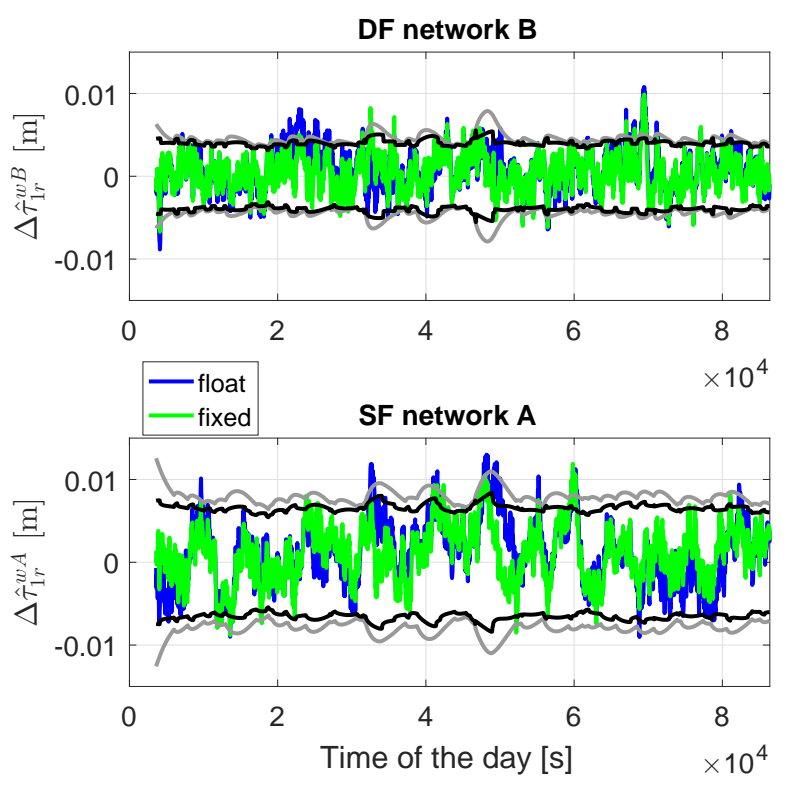

(b) CUT0-STIG (17 km)

(a) CUT0-SLTP (3 km)

Fig. 5. Estimated rZWDs deviated from their true values in simulations for (a) baseline CUT0-SLTP with a length of around $3 \mathrm{~km}$ and (b) baseline CUT0-STIG with a length of around $17 \mathrm{~km}$. The blue and green lines represent the deviations in ambiguity-float and -fixed cases, respectively, and the gray and black lines construct the $95 \%$ formal confidence intervals for ambiguity-float and -fixed cases, respectively. The process noise of ZWDs was set to be $0.1 \mathrm{~mm} / \sqrt{\mathrm{s}}$ by simulation. The parameters $l_{\max }$ (Eq. A.2) and $\sigma_{\iota}$ (Eq. A.3) for ionosphere-weighted model in SF network A were set to be $30 \mathrm{~km}$ and $5 \mathrm{~cm}$, respectively. The first hour is considered as the filter initialization phase and is therefore not included in the plots. The fixed and float solutions thus do not show significant differences

Table 4. Empirical standard deviations of $\Delta \hat{\tau}_{1 r}^{w A}$ (Eq. 17) and $\Delta \hat{\tau}_{1 r}^{w B}$ (Eq. 18) and the average formal standard deviations of $\hat{\tau}_{1 r}^{w A}$ (Eq. 10) and $\hat{\tau}_{1 r}^{w B}$ (Eq. 14) in ambiguity-float and -fixed cases

\begin{tabular}{c|c|c|c|c}
\hline Network & \multicolumn{2}{|c|}{ CUT0 - SLTP $(3 \mathrm{~km})$} & CUT0 - STIG $(17 \mathrm{~km})$ \\
\hline & Formal $[\mathrm{mm}]$ & Empirical $[\mathrm{mm}]$ & Formal $[\mathrm{mm}]$ & Empirical $[\mathrm{mm}]$ \\
\hline & \multicolumn{5}{|c}{ Ambiguity-float } \\
\hline Network A (SF) & 1.7 & 1.7 & 4.1 & 4.0 \\
\hline Network B (DF) & 2.3 & 2.3 & 2.3 & 2.5 \\
\hline & \multicolumn{5}{|c}{ Ambiguity-fixed } \\
\hline Network A (SF) & 1.5 & 1.6 & 3.4 & 3.5 \\
\hline Network B (DF) & 2.1 & 2.1 & 2.1 & 2.2 \\
\hline
\end{tabular}


fixed solutions. This is why the differences between the ambiguity-float and -fixed solutions are marginal. For the long baseline CUT0-STIG of $17 \mathrm{~km}$ (Figure 5b), the DF network B delivers more precise ZWDs than the SF network A, while the situation changes for the short baseline CUT0-SLTP of $3 \mathrm{~km}$ (Figure 5a). The precision of the rZWDs does not vary much with the baseline length for the DF network B, however, for the SF network A, it is improved by a factor of more than 2 when decreasing the baseline length from 17 to $3 \mathrm{~km}$. For short baselines, the ZWD estimates from the SF network A benefit from the strong spatial correlation of the ionospheric delays and become thus more precise. By changing the parameter settings in the ionosphere-weighted model of the SF network A, i.e., the parameters $l_{\max }$ (Eq. A.2) and $\sigma_{\iota}$ (Eq. A.3), the correlation between the ionospheric delays of different stations and the weights of the spatial ionospheric constraints will change accordingly. This leads also directly to changes in the precision of the ZWD estimates. Figure 6 shows the changes of the ionosphere correlation $q_{i j}$ (Eq. A.2) with the inter-station distance $l_{i j}$ under different pre-defined $l_{\text {max }}$. With increasing parameter $l_{\max }$, the ionosphere correlation for stations with the same distance increases based on Eq. A.2, which leads thus also to decreasing standard deviations of the ionospheric constraints (Eq. A.3). Figure 7 shows the standard deviations of the ionospheric constraints (Eq. A.3) for baselines CUT0-SLTP and CUT0-STIG assuming an mean elevation angle $\bar{e}^{s}$ of 50 degrees. The standard deviations increase with the increasing parameter $\sigma_{\iota}$ and the decreasing betweenstation correlation, i.e., the decreasing $l_{\max }$. According to Dach et al. (2015), under an elevation mask of 10 degrees and quiet ionospheric condition with a Total Electron Content (TEC) value of 10 TECU (TEC Units), ionospheric gradient of around $1.5 \mathrm{~mm} / \mathrm{km}$ can be expected for L1 solutions, which correspond to between-station ionospheric differences of around $4.5 \mathrm{~mm}$ and $2.5 \mathrm{~cm}$ for baselines CUT0-SLTP and CUT0-STIG, respectively. Comparing with the values shown in Figure 7 , using e.g. the $l_{\max }$ of $30 \mathrm{~km}$ (the green lines), $\sigma_{\iota}$ smaller than $4 \mathrm{~cm}$ should be used under quiet ionosphere conditions.

Apart from the ionosphere spatial correlation, under different troposphere conditions, different process noise $s_{\tau}$ could be applied to correspond to the ZWD temporal variations. As examples, for baselines CUT0- SLTP and CUT0-STIG, Figure 8 shows the changes of the average formal standard deviations of $\hat{\tau}_{1 r}^{w A}$ (Eq. 10) and $\hat{\tau}_{1 r}^{w B}$ (Eq. 14) with the troposphere process noise $s_{\tau}$ under different ionosphere conditions, i.e., when using different $l_{\max }$ and $\sigma_{\iota}$. The black lines 


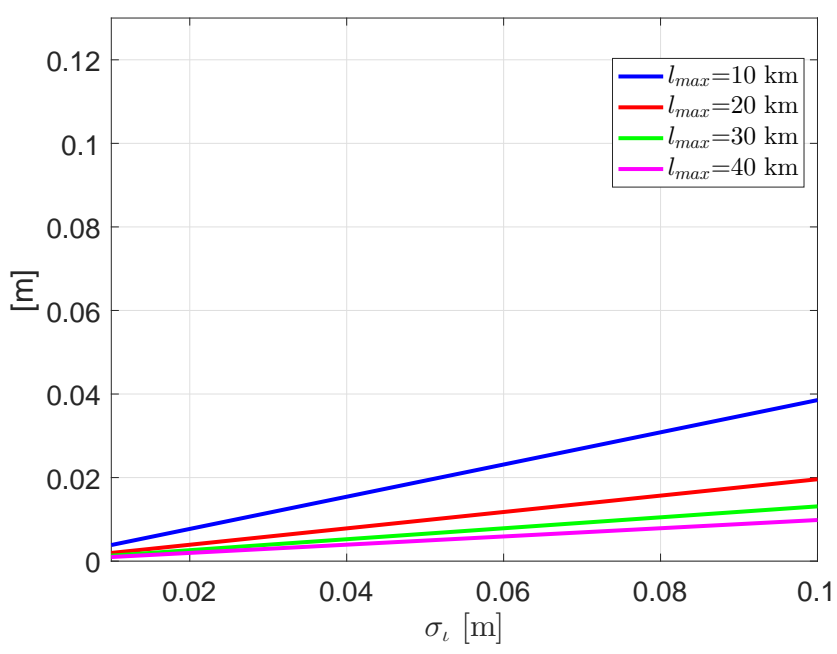

(a) CUT0-SLTP (3 km)

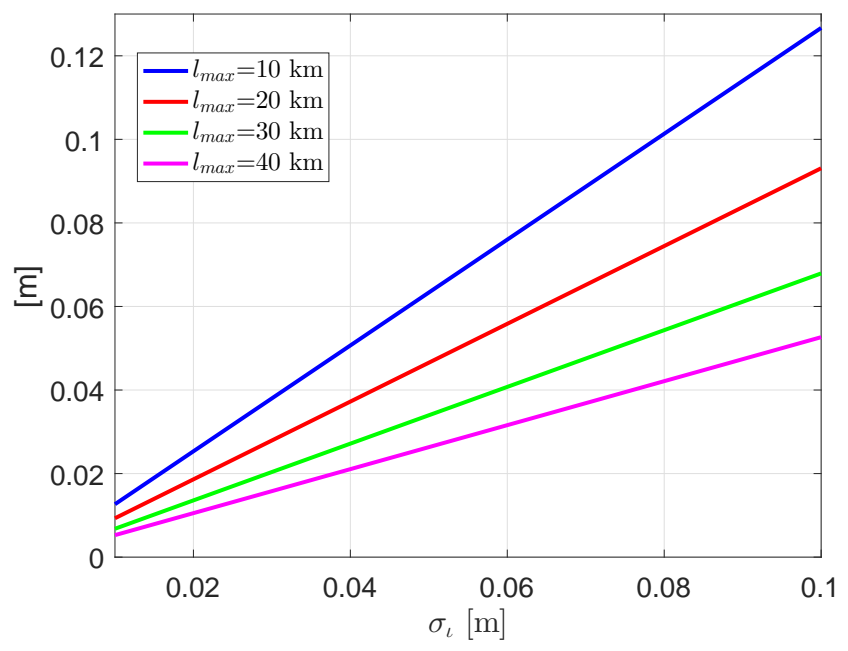

(b) CUT0-STIG $(17 \mathrm{~km})$

Fig. 7. Standard deviations of the ionospheric spatial constraints (Eq. A.3) for (a) baseline CUT0-SLTP of around $3 \mathrm{~km}$ and (b) baseline CUT0-STIG of around $17 \mathrm{~km}$. The mean elevation angle $\bar{e}^{s}$ (Eq. A.3) was assumed to be 50 degrees

represent the DF solutions from network B applying the ionosphere-float model.

From Figure 8 we see that the average formal standard deviation of the rZWDs increases with the increasing process noise of the ZTDs $s_{\tau}$ for both type of solutions. During stable troposphere conditions when small $s_{\tau}$ is applied in the dynamic model of ZTDs, improved precision of the rZWDs can be expected due to the increasing strength of the model. The weights of the ionosphere spatial constraints increase with the decreasing $\sigma_{\iota}$ and the increasing between-station correlation, i.e., the increasing $l_{\max }$. From Figure 8 it can also be observed that strong ionosphere spatial constraints increase the strength of the model and largely improve the precision of the rZWDs computed in the SF network A. As shown in Figure 8a and d, with $\sigma_{\iota}$ of $1 \mathrm{~cm}$ and $l_{\max }$ larger than $20 \mathrm{~km}$, the SF network A could deliver more precise rZWDs than the DF network B even for the long baseline CUT0-STIG of $17 \mathrm{~km}$. As mentioned before, under quiet ionospheric conditions applying e.g. $l_{\max }$ of $30 \mathrm{~km}$ and $\sigma_{\iota}$ smaller than $4 \mathrm{~cm}$, we can at least expect more precise rZWDs from the SF network A for the short baseline CUT0-SLTP of $3 \mathrm{~km}$.

For a more detailed analysis and a better visualization, another network station located at different places within the region of the SF network A (Figure 1) with varying distance to the reference station CUT0 was added to the present networks. The latitude and longitude of the virtual station varies from $32^{\circ} 03^{\prime} 00^{\prime \prime} \mathrm{S}$ to $31^{\circ} 48^{\prime} 00^{\prime \prime} \mathrm{S}$ with a step of $1^{\prime}$ and from $115^{\circ} 45^{\prime} 00^{\prime \prime} \mathrm{E}$ to $31^{\circ} 54^{\prime} 00^{\prime \prime} \mathrm{E}$ with a step of $1^{\prime}$, respectively. The ionosphere-weighted model with $l_{\max }$ of $30 \mathrm{~km}$ and $\sigma_{\iota}$ of $5 \mathrm{~cm}$ was applied to the SF network A and the process noise of the ZTDs $s_{\tau}$ was set to be $0.1 \mathrm{~mm} / \sqrt{\mathrm{s}}$. The mean formal standard deviations of the rZWDs between the virtual station and the reference station CUT0 are illustrated in Figure 9 for the SF network A with partial ambiguity resolution enabled.

From Figure 9 we see that for the SF network A the precision of the rZWDs is related to the baseline length. For the DF network B, the mean formal precision of the rZWDs amounts to around $2.1 \mathrm{~mm}$ and does not vary much, when the location of the virtual station changes within the region shown in Figure 9. For virtual stations located near the reference station CUT0, with a distance within around $8 \mathrm{~km}$, the rZWDs obtained from the SF network A have better or almost the same precision compared to those obtained from the DF network B. When stronger 


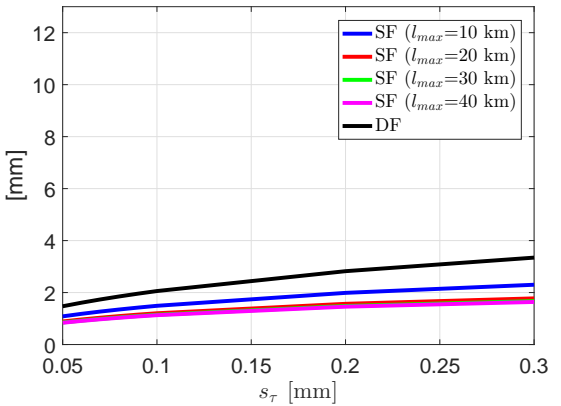

(a) CUT0-SLTP $\left(\sigma_{\iota}=1 \mathrm{~cm}\right)$

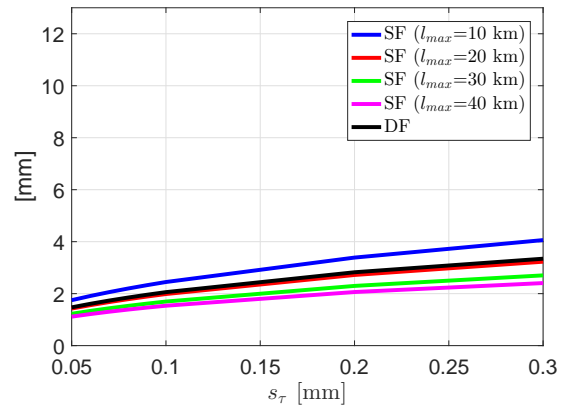

(d) CUT0-STIG $\left(\sigma_{\iota}=1 \mathrm{~cm}\right)$

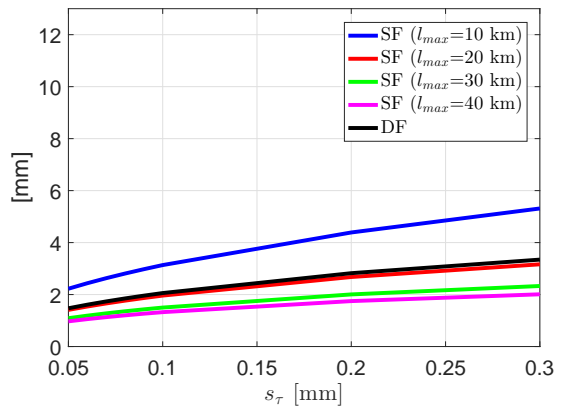

(b) CUT0-SLTP $\left(\sigma_{\iota}=5 \mathrm{~cm}\right)$

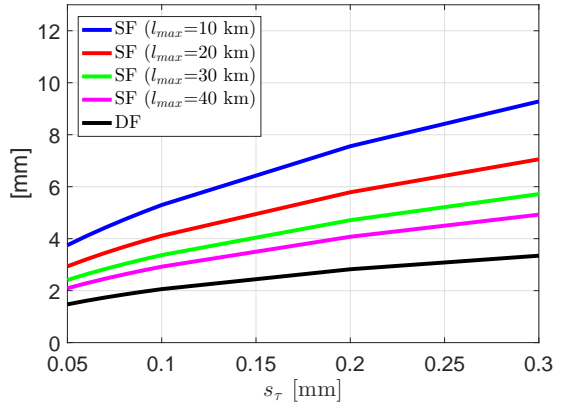

(e) CUT0-STIG $\left(\sigma_{\iota}=5 \mathrm{~cm}\right)$

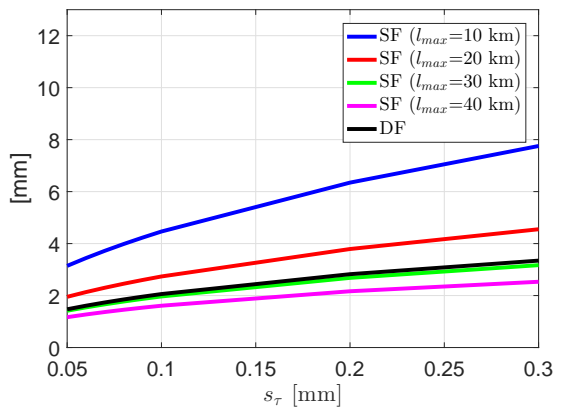

(c) CUT0-SLTP $\left(\sigma_{\iota}=10 \mathrm{~cm}\right)$

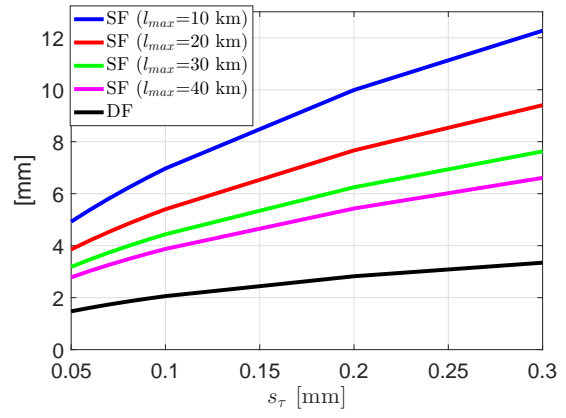

(f) CUT0-STIG $\left(\sigma_{\iota}=10 \mathrm{~cm}\right)$

Fig. 8. Average formal standard deviations of the rZWDs for (a, b, c) baseline CUT0-SLTP of around $3 \mathrm{~km}$ and (d, e, f) baseline CUT0-STIG of around $17 \mathrm{~km}$. The parameter $\sigma_{\iota}$ (Eq. A.3) was set to be (a, d) $1 \mathrm{~cm},(\mathrm{~b}, \mathrm{e})$ $5 \mathrm{~cm}$ and (c, f) $10 \mathrm{~cm}$, respectively. Partial ambiguity resolution was enabled with a pre-defined success rate of 99.9\%. For the SF network A and the DF network B, ionosphere-weighted and -float models were applied in the processing, respectively

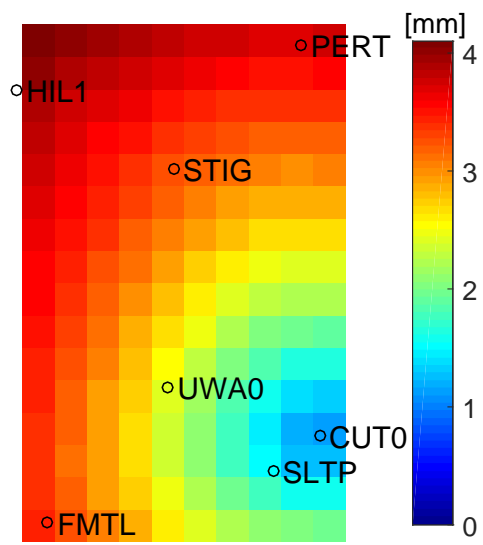

Fig. 9. Mean formal standard deviations of the rZWDs between the virtual station and the reference station CUT0 for the SF network A in ambiguity-fixed case. Ionosphere-weighted model was applied when processing the SF network A with $l_{\max }$ (Eq. A.2) of $30 \mathrm{~km}$ and $\sigma_{\iota}$ (Eq. A.3) of $5 \mathrm{~cm}$. The process noise of the ZWDs was set to be $0.1 \mathrm{~mm} / \sqrt{\mathrm{s}}$ for both networks 


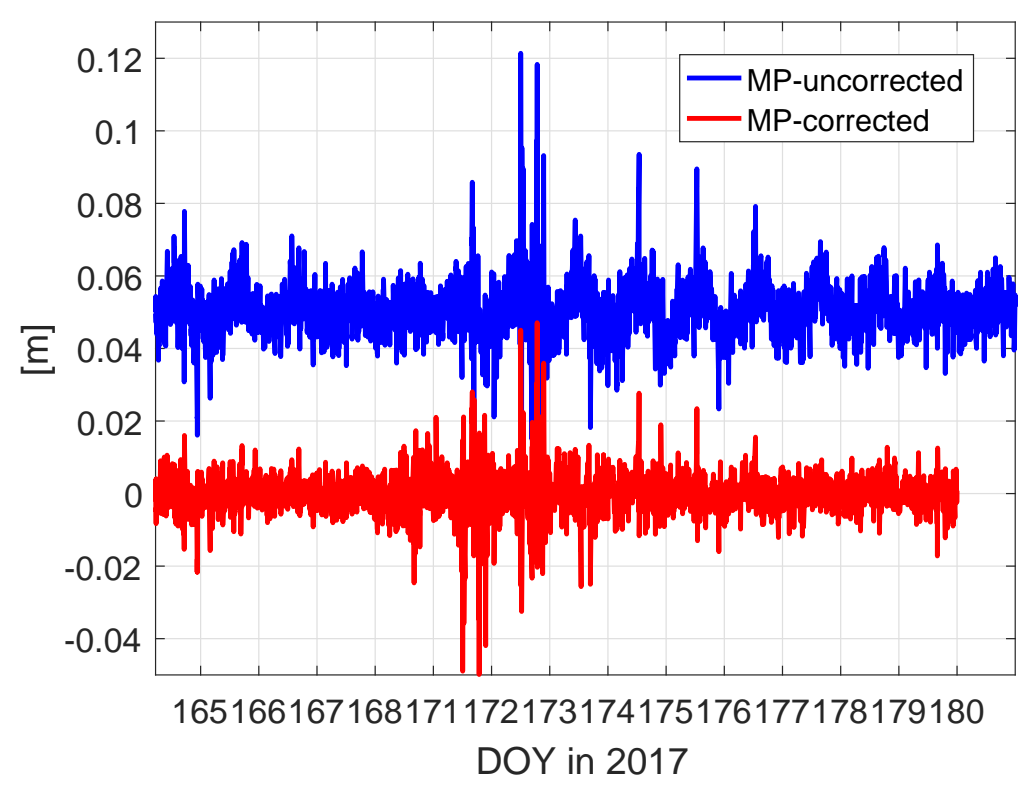

Fig. 10. Differences of the rZWDs estimated in the SF network A and the DF network B for multipath-uncorrected (blue) and multipath-corrected (red) cases. The baseline CUT0-SLTP was used for the plot. The y-values of the blue time series are shifted by $5 \mathrm{~cm}$ for reason of visualization

spatial ionospheric constraints, i.e., larger $l_{\max }$ and smaller $\sigma_{\iota}$, are allowed to be applied to the $\mathrm{SF}$ network A, this distance is expected to be longer.

\subsection{Real data}

Apart from the simulations, we also computed the rZWDs for baselines CUT0-SLTP (3 km) and CUT0-STIG $(17 \mathrm{~km})$ using real GPS data from both networks. The processing was performed on a daily basis except for DOY 164, when the data of the station UWA0 (Figure 1) was only available from 5:11:30 in GPS Time (GPST) and the processing of the SF network A was also started from this time point. Based on the TEC maps provided by SWS (2017), the ionospheric condition is observed to be quiet for the the SF network A during the tested days, i.e., with TEC below or around 10 TECU. Assuming that the ionospheric behaviour is not exactly known before the processing, an ionosphere-weighted model with medium strength was applied with $\sigma_{\iota}$ and $l_{\max }$ of $5 \mathrm{~cm}$ and $30 \mathrm{~km}$. The $\tau_{r}$ was temporally linked with a process noise of $0.1 \mathrm{~mm} / \sqrt{\mathrm{s}}$. Since the true ZWDs are unknown, in this section, we only compare the differences between the SF and DF solutions for both baselines.

The blue time series in Figure 10 shows the differences between the rZWDs estimated in the SF network A and the DF network B, denoted as $\delta \tau_{1 r}^{w}$, for baseline CUT0-SLTP. The y-values of the blue time series are shifted by $5 \mathrm{~cm}$ for reason of visualization. Partial ambiguity resolution was enabled with a pre-defined success rate of $99.9 \%$. Due to the influences of the multipath effects, daily repeated systematic effects can be observed in the blue time series. Assuming that the GPS satellite constellation repeats after $23 \mathrm{~h} 56 \mathrm{~min}$, we also computed the day-to-day differences of the $\delta \tau_{1 r}^{w}$ to mitigate the multipath effects. The day-to-day differences of the $\delta \tau_{1 r}^{w}$ were divided by $\sqrt{2}$ to obtain an approximate pattern of the daily differences of the solutions from both networks (see the red time series in Figure 10), assuming that the ZWDs of two consecutive days are uncorrelated. The missing part between DOY 180 and 181 in the red line is caused by the forming of day-to-day differences. 


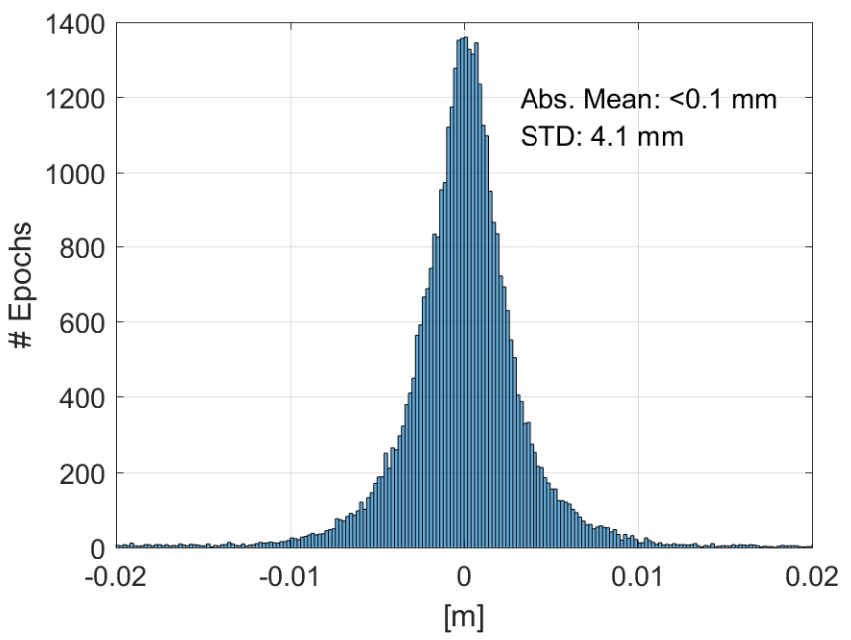

(a) CUT0-SLTP $(3 \mathrm{~km})$

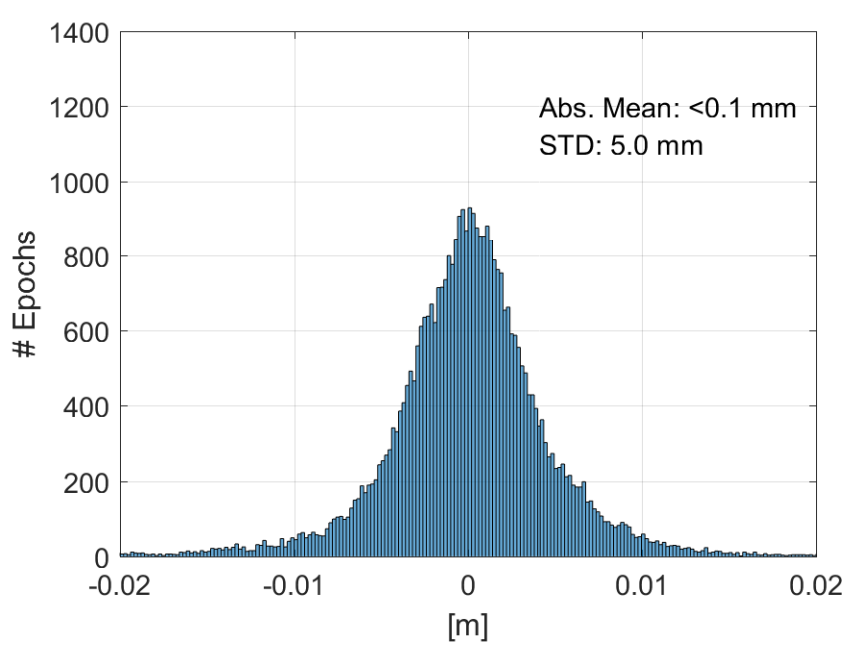

(b) CUT0-STIG $(17 \mathrm{~km})$

Fig. 11. Histograms of the day-to-day differences (divided by $\sqrt{2}$ ) between the rZWDs estimated in the SF network A and DF network B for (a) baseline CUT0-SLTP (3 km) and (b) baseline CUT0-STIG (17 km). Partial ambiguity resolution was enabled with a pre-defined success rate of $99.9 \%$

Table 5. Statistics of the day-to-day differences (divided by $\sqrt{2}$ ) between the rZWDs estimated in the SF network A and DF network B

\begin{tabular}{c|c|c|c|c}
\hline Baseline & Within $\pm 5 \mathrm{~mm}[\%]$ & Within $\pm 1 \mathrm{~cm}[\%]$ & Absolute Mean $[\mathrm{mm}]$ & STD $[\mathrm{mm}]$ \\
\hline & \multicolumn{5}{|c}{ Ambiguity-fixed } \\
\hline CUT0-SLTP $(3 \mathrm{~km})$ & 88.5 & 97.5 & $<0.1$ & 4.1 \\
\hline CUT0-STIG $(17 \mathrm{~km})$ & 78.9 & 95.1 & $<0.1$ & 5.0 \\
\hline & \multicolumn{4}{|c}{ Ambiguity-float } \\
\hline CUT0-SLTP $(3 \mathrm{~km})$ & 69.3 & 92.3 & $<0.1$ & 6.0 \\
\hline CUT0-STIG $(17 \mathrm{~km})$ & 65.7 & 91.2 & $<0.1$ & 6.1 \\
\hline
\end{tabular}

Figure 11 shows the histograms of the day-to-day differences divided by $\sqrt{2}$ (the red time series in Figure 10) between the rZWDs estimated from networks A and B for the ambiguityfixed case. As shown in Table 5, for both baselines over 90\% and 95\% of the differences are within $\pm 1 \mathrm{~cm}$ in ambiguity-float and -fixed cases, respectively.

\section{Conclusions}

This contribution investigates the accuracy of rZWDs based on simulated and real GPS data. Two approaches were used and compared with respect to the resulted between-receiver rZWDs. The first approach utilizes the dual-frequency GPS observations from a large-scale CORS network with the ionospheric delays estimated independently for each station, while the second approach only makes use of the single-frequency GPS signals from a small network, however, with consideration of the spatial ionospheric constraints. In both approaches, the processing was performed on the undifferenced and uncombined level with the estimable parameters formed based on $S$ system theory. All information was thus kept in the observation equations and possibilities of modelling parameters were not sacrificed by forming differences or combinations. 
Based on the simulated data with different ZWD temporal variations and under different ionospheric conditions, it was found that the small-scale SF network (in this study with the longest baseline of $30 \mathrm{~km}$ ) could generate more precise rZWDs than the large-scale DF network (in this study with the longest baseline of $757 \mathrm{~km}$ ) under quiet ionospheric conditions, when strong between-station correlation and large weights can be applied in the ionosphere-weighted model for the SF network. This is especially true for short baselines. Using real GPS data, the differences of the rZWDs were computed using the data from the SF and DF networks. To mitigate the multipath influences, the day-to-day differences were generated and divided by $\sqrt{2}$ to obtain an approximate pattern of the multipath-corrected differences between the two sets of solutions. For both of the tested baselines of 3 and $17 \mathrm{~km}$, above $90 \%$ and $95 \%$ of the differences were within $\pm 1 \mathrm{~cm}$ in ambiguity-float and -fixed cases, respectively. Making use of the spatial correlation of the ionospheric delays, a small SF network could thus deliver rZWDs with comparable accuracy to that of a large-scale DF network.

\section{Acknowledgments}

We would like to thank the IGS for providing the final orbit products, which were obtained through the online archives of the Crustal Dynamics Data Information System (CDDIS), NASA Goddard Space Flight Center, Greenbelt, MD, USA. ftp://cddis.gsfc.nasa.gov/pub/gnss/products/. We would also like to thank Geoscience Australia (GA) and RTKnetwest for providing the GNSS observation data, and Septentrio for providing the receiver at station UWA0 as well as Dr. Sascha Schediwy from the University of Western Australia for hosting this station. Thanks are also given to Australian Bureau of Meteorology, Space Weather Services for putting the TEC map on the server. The station coordinates were processed via the online GPS processing Service AUSPOS provided by GA, and the rainfall data were collected from Bureau of Meteorology. In addition to that, we would also like to thank our colleagues in the GNSS Research Centre, Curtin University, for their development of the Curtin PPP-RTK Software. PJG Teunissen is recipient of an Australian Research Council (ARC) Federation Fellowship (project number FF0883188).

\section{References}

\section{References}

AUSPOS (2018) AUSPOS - Online GPS Processing Service, Geoscience Australia. Processed in January 2018, available at http://www.ga.gov.au/scientific-topics/ positioning-navigation/geodesy/auspos

Baarda W (1981) S-transformations and criterion matrices. In: Publications on geodesy, vol. 5, no. 1, second revised edition, Netherlands Geodetic Commission, Delft, the Netherlands, ISBN-10:9061322189

Barindelli S, Realini E, Venuti G, et al. (2018) Detection of water vapor time variations associated with heavy rain in northern Italy by geodetic and low-cost GNSS receivers. Earth, Planets and Space 70:28. doi:10.1186/s40623-018-0795-7

Bevis M, Businger S, Herring TA, et al. (1992) GPS meteorology: Remote sensing of atmospheric water vapor using the global positioning system. Journal of Geophysical Research: Atmospheres 97(D14):15787-15801. doi:10.1029/92JD01517 
Bock O, Willis P, Wang J, et al. (2014) A high-quality, homogenized, global, long-term (19932008) DORIS precipitable water data set for climate monitoring and model verification. Journal of Geophysical Research: Atmospheres 119(12):7209-7230. doi:10.1002/2013JD021124

Bureau of Meteorology (2018) Daily rainfall data. The Bureau of Meteorology, Australia's national weather, climate and water agency. Accessed in January 2018 at http: //www. bom.gov . $\mathrm{au}$

Coster AJ, Niell AE, Solheim FS, et al. (1996) Measurements of Precipitable Water Vapor by GPS, Radiosondes, and a Microwave Water Vapor Radiometer. In Proc. ION GPS 1996, Kansas City, MO, September 1996, pp. 625-634

Curtin GNSS Research Centre (2018) Observation data. GNSS Research Centre, Curtin University. Accessed in January 2018 at http://saegnss2.curtin.edu/ldc/

Dach R, Dietrich R (2000) Influence of the ocean loading effect on GPS derived precipitable water vapor. Geophysical Research Letters 27(18):2953-2956. doi:10.1029/1999GL010970

Dach R, Lutz S, Walser P, et al. (2015) Bernese GNSS Software Version 5.2. User manual, Astronomical Institute, University of Bern, Bern Open Publishing. doi:10.7892/boris.72297

Deng Z, Bender M, Zus F, Ge M, Dick G, Ramatschi M, Wickert J, Löhnert U, Schön S (2011) Validation of tropospheric slant path delays derived from single and dual frequency GPS receivers. Radio Science 46(6):RS6007. doi:10.1029/2011RS004687

Ding W, Teferle FN, Kazmierski K, et al. (2017) An evaluation of real-time troposphere estimation based on GNSS Precise Point Positioning. Journal of Geophysical Research: Atmospheres 122(5):2779-2790. doi:10.1002/2016JD025727

Dousa J (2001) The impact of ultra-rapid orbits on precipitable water vapor estimation using a ground GPS network. Physics and Chemistry of the Earth, Part A: Solid Earth and Geodesy 26(6-8):393-398. doi:10.1016/S1464-1895(01)00072-2

Dow JM, Neilan RE, Rizos C (2009) The International GNSS Service in a changing landscape of Global Navigation Satellite Systems. J Geod 83(3-4):191-198. doi:10.1007/s00190-008-0300-3

Figurski M, Nykiel G (2017) Investigation of the impact of ITRF2014/IGS14 on the positions of the reference stations in Europe. Acta Geodyn Geomater, Vol. 14, No. 4(188):401-410. doi:10.13168/AGG.2017.0021

Ge M, Calais E, Haase J (2002) Sensitivity of zenith total delay accuracy to GPS orbit errors and implications for near-real-time GPS meteorology. Journal of Geophysical Research: Atmospheres 107(D16):ACL 12-1 - ACL 12-15. doi:10.1029/2001JD001095

Geoscience Australia (2018) Daily observation data. Geoscience Australia. Accessed in January 2018 at ftp://ftp.ga.gov.au/geodesy-outgoing/gnss/data/daily/

Golaszewski P, Stepniak K, Wielgosz P (2017) Zenith tropospheric delay estimates using absolute and relative approaches to GNSS data processing - preliminary results. Geodetic Congress (BGC Geomatics), 2017 Baltic, June 2017, Gdansk, Poland. doi:10.1109/BGC.Geomatics. 2017.79 
Google Earth (2018a) Google Earth imagery (November 5, 2017). Google Earth 7.0.3.8542. Perth, Australia. 31 53'04.40”S, 11556'44.79”E, Eye alt 55.48 km. Data SIO, NOAA, U.S. Navy. NGA. GEBCO, Image (C)2018 DigitalGlobe. https://www.google.com/earth/ [March $22,2018]$

Google Earth (2018b) Google Earth imagery (December 14, 2015). Google Earth 7.0.3.8542.

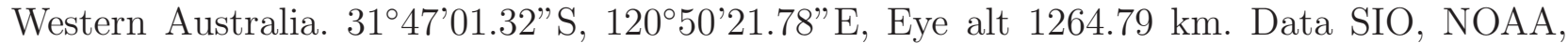
U.S. Navy. NGA. GEBCO, Image Landsat/Copernicus. https://www.google.com/earth/ [March 21, 2018]

Gradinarsky LP, Elgered G (2000) Horizontal gradients in the wet path delay derived from four years of microwave radiometer data. Geophysical Research Letters 27(16):2521-2524. doi:10.1029/2000GL011427

Hofmann-Wellenhof B, Lichtenegger H, Wasle E (2008) GNSS-Global Navigation Satellite Systems: GPS, GLONASS, Galileo, and more. Springer, Vienna. doi:10.1007/978-3-211-73017-1

Ifadis II (1986) The Atmospheric Delay of Radio Waves: Modelling The Elevation Dependence on a Global Scale. Licentiate Thesis, Technical Report No 38L, Chalmers University of Technology, Gothenburg, Sweden

International GNSS Service (IGS) (2018) GNSS final orbit products. NASA CDDIS. Accessed on January 31, 2018 at ftp://cddis.gsfc.nasa.gov/gnss/products/

Nadarajah N, Khodabandeh A, Wang K, et al. (2018) Multi-GNSS PPP-RTK: From Large- to Small-Scale Networks. Sensors 18(4):1078. doi:10.3390/s18041078

Niell AE, Coster AJ, Solheim FS, et al. (2001) Comparison of Measurements of Atmospheric Wet Delay by Radiosonde, Water Vapor Radiometer, GPS, and VLBI. Journal of Atmospheric and Oceanic Technology 18(6):830-850. doi:10.1175/1520-0426(2001)018〈0830:COMOAW $\rangle 2$. $0 . \mathrm{CO} ; 2$

Noll CE (2010) The Crustal Dynamics Data Information System: A resource to support scientific analysis using space geodesy. Adv Space Res 45(12): 1421-1440. doi:10.1016/j.asr.2010.01.018

Odijk D (2002) Fast precise GPS positioning in the presence of ionospheric delays. Ph.D. thesis, Delft University of Technology, Delft, the Netherlands

Odijk D, Khodabandeh A, Nadarajah N, et al. (2017) PPP-RTK by means of S-system theory: Australian network and user demonstration. Journal of Spatial Science 62(1):3-27. doi:10. 1080/14498596.2016.1261373

Odijk D, Zhang B, Khodabandeh A, et al. (2016) On the estimability of parameters in undifferenced, uncombined GNSS network and PPP-RTK user models by means of S-system theory. J Geod 90(1):15-44. doi:10.1007/s00190-015-0854-9

Paddenburg T (2017) It's official: WA the new Sunshine State, with more hours of light than Queensland. https://www.news.com.au/national/western-australia/ its-official-wa-the-new-sunshine-state-with-more-hours-of-light-than-queensland/ news-story/038cc9e64fb862b6e74439a806dd6c8c. Published on May 7, 2017. Accessed on July 31, 2018 
Resch GM (1984) Water Vapor Radiometry in Geodetic Applications. In: Brunner FK (eds) Geodetic Refraction. Springer, Berlin, Heidelberg. pp. 53-84. doi:10.1007/978-3-642-45583-4_5

Rocken C, Van Hove T, Johnson J, et al. (1995) GPS/STORM - GPS sensing of atmospheric water vapor for meteorology. Journal of Atmospheric and Oceanic Technology 12(3):468-478. doi:10.1175/1520-0426(1995)012<0468:GSOAWV $>2.0 . C O ; 2$

Rothacher M, Beutler G (1998) The role of GPS in the study of global change. Phys Chem Earth 23(9-10):1029-1040. doi:10.1016/S0079-1946(98)00143-8

RTKnetwest (2017) Observation data of stations SLTP and STIP in June 2017, RTKnetwest. Accessed in March 2018 at http://www.rtknetwest.com.au/

Saastamoinen J (1972) Contributions to the theory of atmospheric refraction. Bulletin Géodésique 105(1):279-298. doi:10.1007/BF02521844

SWS (2017) TEC map file, Australian Bureau of Meteorology, Space Weather Services. Accessed on July 31, 2018 at ftp://ftp-out.sws.bom.gov.au/wdc/gnss/data/

Teunissen PJG (1985) Zero Order Design: Generalized Inverses, Adjustment, the Datum Problem and S-Transformations. In: Grafarend EW, Sansò F (eds) Optimization and Design of Geodetic Networks, pp. 11-55, Springer, Berlin, Heidelberg. doi:10.1007/978-3-642-70659-2_3

Teunissen PJG, Khodabandeh A (2015) Review and principles of PPP-RTK methods. Journal of Geodesy 89(3):217-240. doi:10.1007/s00190-014-0771-3

Teunissen PJG, Montenbruck O (Eds.) (2017) Springer Handbook of Global Navigation Satellite Systems. Springer, Cham. doi:10.1007/978-3-319-42928-1

Tregoning P, Boers R, O'Brien D, et al. (1998) Accuracy of absolute precipitable water vapour estimates from GPS observations. Journal of Geophysical Research: Atmospheres 103(D22):28701-28710. doi:10.1029/98JD02516

Wang K, Khodabandeh A, Teunissen PJG (2017) A study on predicting network corrections in PPP-RTK processing. Adv Space Res 60(7):1463-1477. doi:10.1016/j.asr.2017.06.043

Wang K, Khodabandeh A, Teunissen PJG, et al. (2018) Satellite-Clock Modeling in SingleFrequency PPP-RTK Processing. Journal of Surveying Engineering 1144(2):04018003. doi: 10.1061/(ASCE)SU.1943-5428.0000252

Yu C, Li Z, Penna NT (2018) Interferometric synthetic aperture radar atmospheric correction using a GPS-based iterative tropospheric decomposition model. Remote Sensing of Environment 204:109-121. doi:10.1016/j.rse.2017.10.038

\section{Appendix A. Appendix}

For ionosphere-weighted model (Eq. 4):

The auto-correlation matrix of the ionospheric delays in the zenith direction $Q_{r r}$ are given as:

$$
Q_{r r}=\left(\begin{array}{ccc}
q_{11} & \cdots & q_{1 n} \\
\vdots & \ddots & \vdots \\
q_{n 1} & \cdots & q_{n n}
\end{array}\right)
$$


with the cross correlation between station $i$ and $j$ defined with the Gaussian function:

$$
q_{i j}=\exp \left(-\left(\frac{l_{i j}}{l_{\max }}\right)^{2}\right)
$$

where $l_{i j}$ represents the inter-station distance between station $i$ and $j$, and $l_{\text {max }}$ is a pre-defined parameter. Making use of the auto-correlation matrix, the elevation weighting function (Eq. 8) and a pre-defined zenith-referenced between-receiver standard deviation of the ionospheric signals $\sigma_{\iota}$, the variance-covariance matrix of the spatial ionospheric constraints for satellite $s$ is formulated as:

$$
Q_{C T}^{s}=D_{n}^{T} \cdot \frac{1}{\sin ^{2}\left(\bar{e}^{s}\right)} \cdot \frac{\sigma_{\iota}^{2}}{2} \cdot Q_{r r} \cdot D_{n}
$$

where $D_{n}^{T}$ is the differencing operator with $D_{n}^{T}=\left[-e_{n-1}, I_{n-1}\right]$, and $\bar{e}^{s}$ represents the mean elevation angle for satellite $s$. The entire variance-covariance matrix of the spatial ionospheric constraints is the block diagonal matrix of $Q_{C T}^{s}$ with $s=1, \cdots, m$, where $m$ denotes the number of satellites. 